\title{
CONTENIDO DE CARBONO EN UN BOSQUE DE TIERRA FIRME DEL RESGUARDO NONUYA-VILLAZUL, AMAZONIA COLOMBIANA
}

\section{Carbon stock in a unflooded forest of the Nonuya-Villazul indigenous reservation in the Colombian amazon}

\author{
Edwin Paky¹, Fabián Moreno² \& Esteban Álvarez-Dávila³
}

Paky, E., Moreno, F. \& Álvarez-Dávila, E. (2017). Contenido de carbono en un bosque de tierra firme del resguardo Nonuya-Villazul, amazonia colombiana. Colombia Forestal, 20(2), 144-157.

Recepción: 18 de noviembre 2016

\section{Resumen}

La implementación de los programas REDD+ requiere estimaciones precisas del carbono forestal. En este estudio se reporta el contenido de carbono en un bosque de tierra firme del Resguardo Villazul-Nonuya RVN de la Amazonia colombiana, con base en una parcela permanente de 6 ha, y se evalúa su variación espacial y la contribución del sotobosque, dos factores poco considerados. Se encontró una biomasa promedio total de $336.1 \pm 14.0$ t.ha $^{-1}$; el $11.5 \%$ se encuentra en el sotobosque (DAP $1-10 \mathrm{~cm}$ ) y el $88.5 \%$ corresponde a árboles con DAP $\geq 10 \mathrm{~cm}$; los árboles grandes (DAP $\geq 70 \mathrm{~cm})$ son pocos $(4 \pm 1$ por ha) y tienen una baja contribución a la biomasa (6.8\%) en comparación con otros bosques tropicales. La biomasa promedio estimada está dentro del rango reportado para toda la cuenca Amazónica, es superior a la Amazonia Occidental y similar al de Amazonia colombiana. Considerando que el Resguardo VN tiene 208800 ha en bosques de tierra firme, se estimó un promedio total de 31.0 Mt C (95\% IC 29.1 - 32.04). Estos resultados permiten ubicar a los bosques del Resguardo VN en un contexto global y mejorar la precisión de las estimaciones de carbono forestal en la Amazonia colombiana.

Palabras clave: biomasa forestal, cambio climático, monitoreo del bosque, parcela permanente.
Aprobación: 22 de mayo 2017

\begin{abstract}
Implementation of REDD programs requires accurate estimates of forest carbon. This study reports biomass and carbon in a forest of the Nonuya-Villazul indigenous reservation (RVN) in the Colombian Amazon, based on a permanent plot of 6 ha. The local spatial variation of the biomass and the contribution of the undergrowth were evaluated, two factors that are rarely taken into account. A total average biomass of $336.1 \pm 14.0$ t.ha $^{-1}$ was found; $11.5 \%$ is in the understory (DAP $1-10 \mathrm{~cm}$ ) and $88.5 \%$ corresponds to trees with $\mathrm{DBH} \geq 10 \mathrm{~cm}$; large trees (DAP $\geq 70 \mathrm{~cm})$ are few $(4 \pm 1 \mathrm{ha})$ and have a low contribution to total biomass $(6.8 \%)$ compared to other tropical forests. The biomass is within the range reported for the Amazon basin, is superior to the Western Amazon and similar to the Colombian Amazon. Considering that the RVN has 208800 ha in unflooded forests, a total average of $31.0 \mathrm{Mt} \mathrm{C}(95 \% \mathrm{Cl}$ 29.1 - 32.04) was estimated. These results allow to locate the forests of the RVN in a global context and improve the forest carbon estimations in the Colombian Amazon.
\end{abstract}

Keywords: forest biomass, climate change, forest monitoring, permanent plot.

\footnotetext{
Universidad de la Amazonia. Florencia, Caquetá. pakybarbosa@gmail.com

Grupo de Socio Ecosistemas y Clima Sostenible (GSEC), Fundación ConVida, Medellín, Colombia. unuva_famgo@yahoo.com

Escuela ECAPMA, Universidad Nacional Abierta y a Distancia (UNAD Colombia). esalvarez3000@gmail.com. Autor de correspondencia
} 


\section{INTRODUCCIÓN}

En la Amazonia colombiana los resguardos indígenas cubren cerca de 23 millones de hectáreas, la mayor parte cubiertas por bosques (Salinas-Abdala, 2014). Esto implica que las comunidades nativas custodian cerca del 33\% del stock nacional de carbono forestal en Colombia y, por ello, son actores estratégicos para el cumplimiento de los compromisos internacionales de mitigación del cambio climático (García-Arbeláez et al., 2015). Actualmente, los programas REDD+ se consideran la forma más rápida, económica y rentable de hacer frente a las emisiones por deforestación, de promover la conservación y el manejo sostenible del bosque tropical y mejorar las reservas de carbono forestal (Watch, 2013). Sin embargo, la implementación de los REDD+ en territorios indígenas tienen pros y contras; por un lado, representan una oportunidad de recibir ingresos económicos significativos que pueden servir para suplir las necesidades o deseos de las comunidades, pero al tiempo pueden conducir a la usurpación de sus derechos por parte de personas ajenas o a mayores dificultades debido a nuevas limitaciones en el uso del bosque (Larson et al., 2010). En este contexto, es evidente la importancia que las comunidades indígenas estén bien informadas y capacitadas sobre todos los aspectos e implicaciones culturales, políticas y técnicas de los programas REDD+.

Desde 1990 se desarrollan estudios en la región del medio Caquetá (Amazonia colombiana) con la cooperación de las comunidades locales. Gracias a este apoyo se tienen estimaciones previas de la biomasa forestal que varían entre más de 400 t.ha $^{-1}$ (Londoño, 2011) y 207.6 t.ha $^{-1}$ (Asner et al., 2012). De tal manera, es importante contar con nueva información que ayude a reducir esta incertidumbre.

En el presente estudio se caracterizó la variación local en el almacenamiento de carbono en un bosque de tierra firme. Concretamente, se quiso responder a las siguientes preguntas: ¿cuál es la biomasa y el contenido de carbono por hectárea en el bosque? ¿Cómo influye la variación espacial y la inclusión del sotobosque en las estimaciones de biomasa? De acuerdo con estos resultados, ¿qué tanto carbono, potencialmente, almacenan los bosques del resguardo Villazul Nonuya? ¿Cómo se comparan los resultados con estimaciones previas?

Con este estudio se espera contribuir al conocimiento del carbono forestal en los bosques de la Amazonia donde existen aún muchos vacíos y aportar información para la posible implementación de un programa REDD+ en el resguardo.

\section{MATERIALES Y MÉTODOS}

\section{Área de estudio}

Se localiza en predios de la comunidad indígena de Peña Roja, en el resguardo Nonuya de Villa azul de la región de Araracuara, cuenca media del río Caquetá en la Amazonia colombiana (figura 1). La región del medio Caquetá hace parte de la denominada región noroccidental dentro de la gran cuenca del río Amazonas (Mitchard et al., 2014), y corresponde a un bosque húmedo tropical (bh-t) en el sistema de zonas de vida de Holdridge (1982), con temperatura promedio anual de $25.7{ }^{\circ} \mathrm{C}$, precipitación promedio anual de $3059 \mathrm{~mm}$ y una distribución de las Iluvias unimodal sin estación seca; la época de menor precipitación ocurre durante los meses de diciembre a febrero (Londoño, 2011).

En el 2010, por iniciativa de la comunidad indígena del resguardo, se estableció una parcela permanente (coordenadas $-0.66^{\circ} \mathrm{S}$ y $-72.1^{\circ} \mathrm{O}$ ) para el monitoreo de la vegetación de 6 ha $(200$ x 300 m), tomando como base una parcela de 1.8 ha previamente establecida por la fundación Tropenbos en 1990 (Londoño, 2011). La parcela está ubicada en un bosque sin evidencias de intervenciones recientes de origen humano; la fisiografía corresponde al plano sedimentario terciario, o tierra firme, que cubre el $90 \%$ de la Amazonia colombiana con una altitud promedia de $60 \mathrm{~m}$ sobre el nivel medio del río Caquetá. En el área los suelos se clasifican como Ultisoles (Londoño, 2011). 


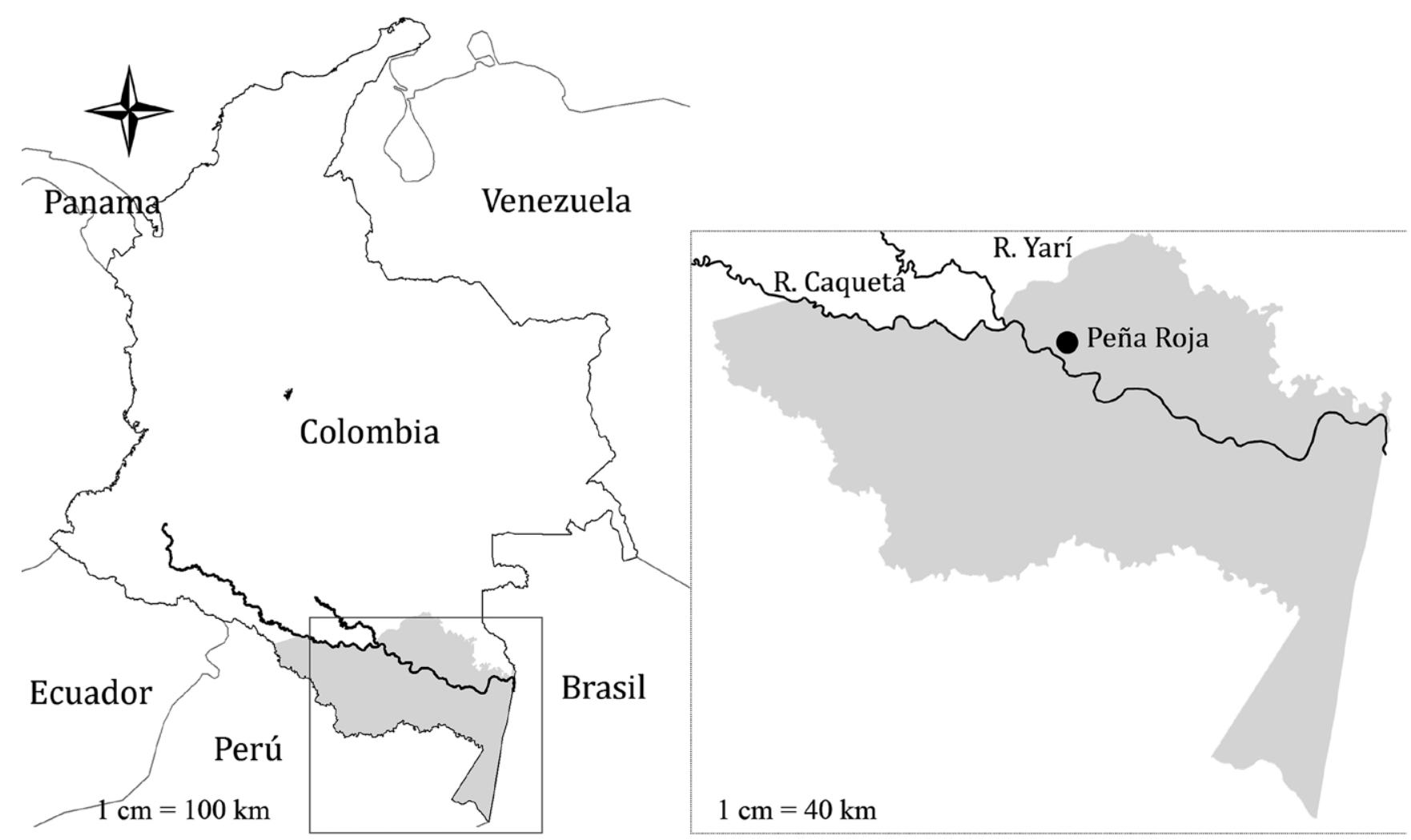

Figura 1. Localización del área de estudio en la región de Araracuara, Amazonia Colombia (arriba).

\section{Métodos}

\section{Trabajo de campo}

Dentro de la parcela de 6 ha se midieron todos los árboles con DAP $\geq 10 \mathrm{~cm}$ y se colectaron muestras botánicas para identificar los árboles, palmas y lianas; la parcela fue subdividida en parcelas de 1 ha y estas a su vez en cuadrantes de 20×20 m; en el centro de cada cuadrante se delimitó una franja de $2 \times 20 \mathrm{~m}$ donde se censaron las plantas del sotobosque (DAP $1-10 \mathrm{~cm}$ ).

\section{Estimación de la biomasa y el carbono}

Para la estimación del carbono el método más aceptado actualmente es el de utilizar modelos alométricos que permiten calcular la biomasa de los árboles individuales registrados en las parcelas de un inventario forestal, a partir de variables predictoras como el diámetro del tronco (DAP $\mathrm{cm}$ ) y la densidad de la madera $\left(\mathrm{Dm} \mathrm{g} \cdot \mathrm{cm}^{-3}\right)$. Para cada parcela se suma la biomasa de los árboles y dependiendo de su área se Ileva a unidades de una hectárea (Chave et al., 2005). Posteriormente, la biomasa es convertida en unidades de carbono multiplicándola por un factor de 0.5 (Eggleston et al., 2006).

Para Colombia se cuentan con modelos nacionales de biomasa para las seis zonas de vida más representativas del país, los cuales son usados para estimar la biomasa de los bosques húmedos de la Amazonia (Álvarez et al., 2012; Phillips et al., 2016; Álvarez-Dávila et al., 2017). Para los árboles con $\mathrm{DAP} \geq 10 \mathrm{~cm}$ se eligió el modelo II.1 de Álvarez et al. (2012) que utiliza el DAP y la Dm, porque ha demostrado ser el más adecuado para la región. En el caso de los árboles del sotobosque utilizamos una ecuación no publicada (Rodríguez, 1991; Londoño, 2011 ) que fue construida con árboles pequeños 
entre 4-55 cm DAP en un sitio cercano a la parcela de 6 ha y en la misma unidad fisiográfica. Para las palmas de todos los tamaños a las que se pudiera medir de DAP (se excluyen las acaules) se utilizó el modelo de Goodman et al. (2013) para la familia y para lianas el de Schnitzer et al. (2006) (tabla 1). Para las ecuaciones que usan la densidad de la madera esta fue asignada a cada una de las especies, géneros o familias en la parcela a partir de la base de datos Dryad (Zanne et al., 2009), de acuerdo con Chave et al. (2014).

Se calcularon los promedios y los intervalos de confianza (IC) del 95\% para la biomasa y la densidad de tallos mediante el método bootstraps con 1000 iteraciones, usando las parcelas de 1 ha para proporcionar información sobre la variación espacial en la biomasa (Ngo et al., 2013). Se reportaron estimaciones de biomasa por hectárea a nivel de especies y familias, obtenidas al dividir la biomasa total para cada especie y familia en la parcela por el área total muestreada en hectáreas.

Se evaluó la variación espacial con base en parcelas de 0.25 ha $(50 \times 50 \mathrm{~m})$, considerado el tamaño mínimo representativo para abarcar la variación local en la estructura de los bosques tropicales (Chave et al., 2004) y en parcelas de $20 \mathrm{x}$ $50 \mathrm{~m}$, que corresponde al tamaño usado más comúnmente para estimar la biomasa de los bosques de Amazonia colombiana (Phillips et al., 2011). Para evaluar el efecto de la topografía en cada cuadrante de 20 × 20 m se estimó la biomasa, la pendiente en $\%$ y la altitud promedio sobre el nivel del rio
Caquetá. Posteriormente, se efectuó un análisis de regresión lineal para evaluar si la pendiente, la altitud o ambas explicaban la variación observada en la biomasa. Todos los cálculos se realizaron con Statgraphics XV.I. (Statgraphics Centurion, 2013)

\section{RESULTADOS}

\section{Estimación de la biomasa}

Se estimó una biomasa para individuos con DAP $\geq 10 \mathrm{~cm}$ de 297.6 t.ha $^{-1}$ equivalentes a 148.8 t.ha $^{-1}$ de C (IC 95\% 165.8-176.8); el 99.5\% corresponde a los árboles (tabla 2). En el sotobosque (DAP 1 - 10 $\mathrm{cm}$ ) encontramos un promedio de 7798 tallos.ha $^{-1}$ (de los cuales el $82.5 \%$ fueron árboles, el 14.5\% lianas y el resto palmas) que contenían una biomasa promedio de 38.6 t.ha $^{-1}$ (tabla 2), equivalente al $13 \%$ de la biomasa para árboles por encima de este diámetro. Los árboles en el sotobosque contienen la mayor biomasa $(11.8 \%)$, seguido de las lianas $(1.2 \%)$, mientras que la contribución de las palmas es mucho menor (no se tuvieron en cuenta las palmas acaules). Considerando la biomasa del sotobosque, se estimó una biomasa promedio total de 336.1 t.ha-1 para el bosque de tierra firme estudiado en el resguardo Villazul-Nonuya. La mayor parte de la biomasa en la parcela se encontró en los árboles de 0-70 cm DAP (tabla 2). Se hallaron pocos árboles grandes; 22 por encima de $50 \mathrm{~cm}$ y solo 4 por encima de $70 \mathrm{~cm}$, con una contribución

Tabla 1. Ecuaciones utilizadas para estimar la biomasa. Biom = biomasa $(\mathrm{kg} /$ árbol $), \mathrm{DAP}=$ diámetro $(\mathrm{cm}), \mathrm{At}=$ Altura total $(\mathrm{m}), \mathrm{Dm}=$ densidad de la madera $(\mathrm{g} / \mathrm{cc})$.

\begin{tabular}{ccccc}
\hline & Código del modelo & Tipo de bosque & Modelo para las biomasas del árbol (kg) & Fuente \\
\hline 1 & Árboles DAP $\geq 10 \mathrm{~cm}$ & Bh-T Colombia & $\exp \left(\left(2.406-1.289 * \ln (\mathrm{DAP})+1.169^{*}(\ln (\mathrm{DAP})) 2-\right.\right.$ & \multicolumn{1}{c}{ Álvarez et al. (2012) } \\
2 & Árboles $1-10 \mathrm{~cm}$ & Amazonia Col & $\exp \left(-1.6028+\left(2.4242^{*} \ln (\mathrm{DAP})\right)\right)$ & Rodriguez $(2001)$ \\
3 & Palmas & Amazonia & $\exp (-3.3488+2.7483 * \ln (\mathrm{DAP}))$ & Goodman et al. (2013) \\
4 & Lianas & Amazonia & $\exp (-1.484+2.657 * \ln (\mathrm{DAP}))$ & Schnitzer et al. (2006) \\
\hline
\end{tabular}


relativamente menor a la biomasa total del bosque; $25.1 \%$ con respecto al total en el caso de los mayores a $50 \mathrm{~cm}$ y $6.8 \%$ en el caso de los mayores a $70 \mathrm{~cm}$. Es destacable que en el bosque estudiado la biomasa de los árboles por debajo de $10 \mathrm{~cm}$, fue superior que la biomasa de los árboles grandes con $\mathrm{DAP} \geq 70 \mathrm{~cm}$.

Las 10 especies de árboles más importantes en términos de biomasa representaron el $37.2 \%$ del total (tabla 3). El árbol más abundante en nuestra parcela, Pseudomonotes tropenbosii, representó el 9.9 \%. Las 20 especies con mayor biomasa para toda la amazonia pertenecen a 13 familias
(Fauset et al., 2015), 8 de las cuales están entre las 10 con mayor biomasa en el bosque de tierra firme estudiado (Fabaceae, Lecythidaceae, Sapotaceae, Lauraceae, Vochysiaceae, Apocynaceae, Burseraceae y Moraceae; tabla 3).

\section{Variación local}

La variación en la biomasa total entre parcelas contiguas de 1 ha fue relativamente alta (rango 272.2-322.7 t.ha-1), con un promedio de 297.6 t.ha $^{-1}$. Cuando se considera un tamaño de parcela de 50 × $50 \mathrm{~m}$, la variación se incrementó

Tabla 2. Distribución de la densidad de tallos y de la biomasa en categorías de tamaño. Se presenta el promedio por hectárea (Prom) para la parcela de 6 ha, la desviación estándar (De), los intervalos del 95\% de confianza bootstrapping, inferior (ICi) y superior (ICs) y el \% con respecto al total.

\begin{tabular}{|c|c|c|c|c|c|c|c|c|c|c|}
\hline Rango & Prom & De & $\mathrm{ICi}$ & ICs & $\%$ & Prom & De & ICi & ICs & $\%$ \\
\hline & \multicolumn{5}{|c|}{ Densidad de tallos $\mathrm{n} / \mathrm{ha}$} & \multicolumn{5}{|c|}{ Biomasa t.ha ${ }^{-1}$} \\
\hline $0-10$ & 7182 & 811 & 6612 & 7698 & 89.8 & 38.6 & 8.9 & 32.1 & 44.5 & 11.5 \\
\hline $10-50$ & 794 & 95 & 731 & 857 & 9.9 & 213.4 & 23.5 & 197.5 & 230.2 & 63.5 \\
\hline $10-70$ & 812 & 93 & 751 & 875 & 10.2 & 274.8 & 22.9 & 260.1 & 291.2 & 81.8 \\
\hline$>10$ & 816 & 92 & 750 & 871 & 10.2 & 297.6 & 18.8 & 284.4 & 310.4 & 88.5 \\
\hline$>50$ & 22 & 5 & 18 & 25 & 0.3 & 84.2 & 22.0 & 68.1 & 98.8 & 25.1 \\
\hline$>70$ & 4 & 1 & 3 & 5 & 0.0 & 22.8 & 10.3 & 16.6 & 30.0 & 6.8 \\
\hline Total & 7998 & 804 & 7460 & 8497 & & 336.1 & 14.0 & 326.0 & 344.5 & \\
\hline
\end{tabular}

Tabla 3. 10 especies y familias con mayor biomasa $\left(\mathrm{t} . h a^{-1}\right)$ y su contribución (\%) a la biomasa total, para árboles con DAP $\geq 10 \mathrm{~cm}$.

\begin{tabular}{|c|c|c|c|c|c|c|}
\hline Especie & Familia & Biom t.ha' ${ }^{-1}$ & $\%$ & Familia & Biom t.ha-1 & $\%$ \\
\hline Pseudomonotes tropenbosi & Dipterocarpaceae & 29.3 & 9.9 & Fabaceae & 68.8 & 23.1 \\
\hline Swartzia amplifolia & Fabaceae & 25.3 & 8.5 & Dipterocarpaceae & 29.3 & 9.9 \\
\hline Eschweilera punctata & Lecythidaceae & 10.4 & 3.5 & Lecythidaceae & 23.9 & 8.0 \\
\hline Monopteryx uаuсu & Fabaceae & 10.0 & 3.4 & Sapotaceae & 18.6 & 6.3 \\
\hline Aspidosperma excelsum & Apocynaceae & 8.2 & 2.7 & Lauraceae & 15.6 & 5.2 \\
\hline Erisma splendens & Vochysiaceae & 6.2 & 2.1 & Vochysiaceae & 14.7 & 4.9 \\
\hline Scleronema micranthum & Malvaceae & 5.4 & 1.8 & Apocynaceae & 14.6 & 4.9 \\
\hline Brosimum rubescens & Moraceae & 4.8 & 1.6 & Myristicaceae & 13.0 & 4.4 \\
\hline Clathrotropis macrocarpa & Fabaceae & 4.7 & 1.6 & Burseraceae & 12.0 & 4.0 \\
\hline Eschweilera laevicarpa & Lecythidaceae & 4.3 & 1.4 & Moraceae & 10.3 & 3.5 \\
\hline Total & & 108.5 & 36.5 & Total & 220.8 & 74.2 \\
\hline
\end{tabular}


considerablemente, entre 224.2 a 419.6 t.ha ${ }^{-1}$; aún más para parcelas de $20 \times 50 \mathrm{~m}$ (rango 156.5 a 604.7 t.ha $\left.^{-1}\right)$. De acuerdo con la figura 2, no se observó ningún patrón explícito de autocorrelación espacial para los diferentes tamaños de parcela considerados, ni tampoco una relación entre la biomasa con la pendiente o la elevación. El análisis de regresión entre la biomasa en parcelas de 20×20 m no mostró ningún efecto de la pendiente $\left(R^{2}=2.2 \% ; F=3.40 ; P=0.0671\right)$ o de la elevación $\left(R^{2}=0.5 ; F=0.64 ; P=0.425\right)$, ni de las dos variables combinadas $\left(R^{2}=0 \% ; F=0.47 ; P=0.629\right)$.

\section{Contenido de carbono de los bosques del resguardo Villazul}

Considerando que el resguardo Villazul Nonuya tienen un área total de 260933 ha, y asumiendo que el bosque de tierra firme corresponde significativamente a la mayor proporción del resguardo, la mejor estimación de la biomasa con el promedio y los IC para 1 ha es de 88.5 millones de toneladas (95\% IC 84.6-92.4) lo que equivale a un contenido total de carbono de $44.3 \mathrm{t}(95 \%$ IC 42.9-45.6).
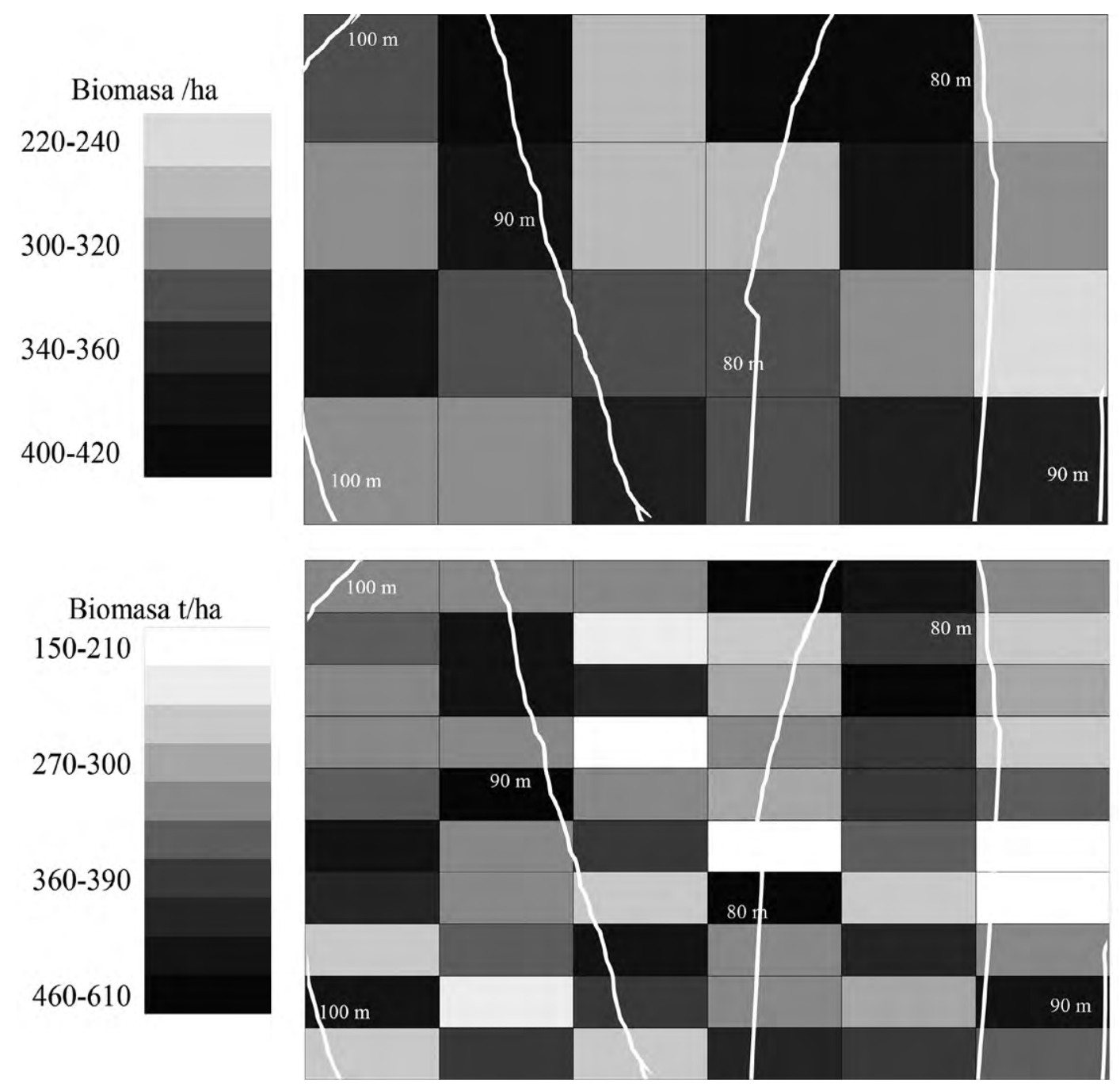

Figura 2. Variación espacial en la biomasa total t.ha-1 en la parcela de 6 ha en Peña Roja, mostrando la variación en la altura de la parcela con respecto al nivel medio del río Caquetá (líneas blancas). Los tonos de grises indican la variación en biomasa en subparcelas de 50 × 50 m (arriba) de 20 × 50 m (abajo). 


\section{DISCUSIÓN}

En comparación con otros bosques tropicales de tierras bajas, el valor promedio de biomasa encontrado en el presente estudio para árboles con DAP $\geq 10 \mathrm{~cm}$ es similar al promedio para las Américas (287.9 Mg.ha-1) (Slik et al., 2013), pero más bajo que los reportes promedio para Asia (393.24), África (393.3) (Slik et al., 2013) y Australia (513.6 Mg.ha-1) (Bradford et al., 2014; Murphy et al., 2013). Aunque la Amazonia colombiana alberga la mayor extensión de bosques del país, los estudios sobre la biomasa forestal y el almacenamiento de carbono en campo son pocos (Phillips et al., 2016; Álvarez-Dávila et al., 2017). Aun así, existen mapas elaborados a partir de sensores remotos a nivel nacional (Anaya et al., 2009; Galindo et al. 2011), regional, para una porción de la Amazonia (Asner et al., 2012), y mapas pantropicales de biomasa (Baccini et al., 2012; Saatchi et al., 2011), de donde es posible extraer información para el sitio de estudio. La comparación de la biomasa para árboles con DAP $\geq 10 \mathrm{~cm}$ (figura 3) muestra que las estimaciones previas para Colombia son divergentes con

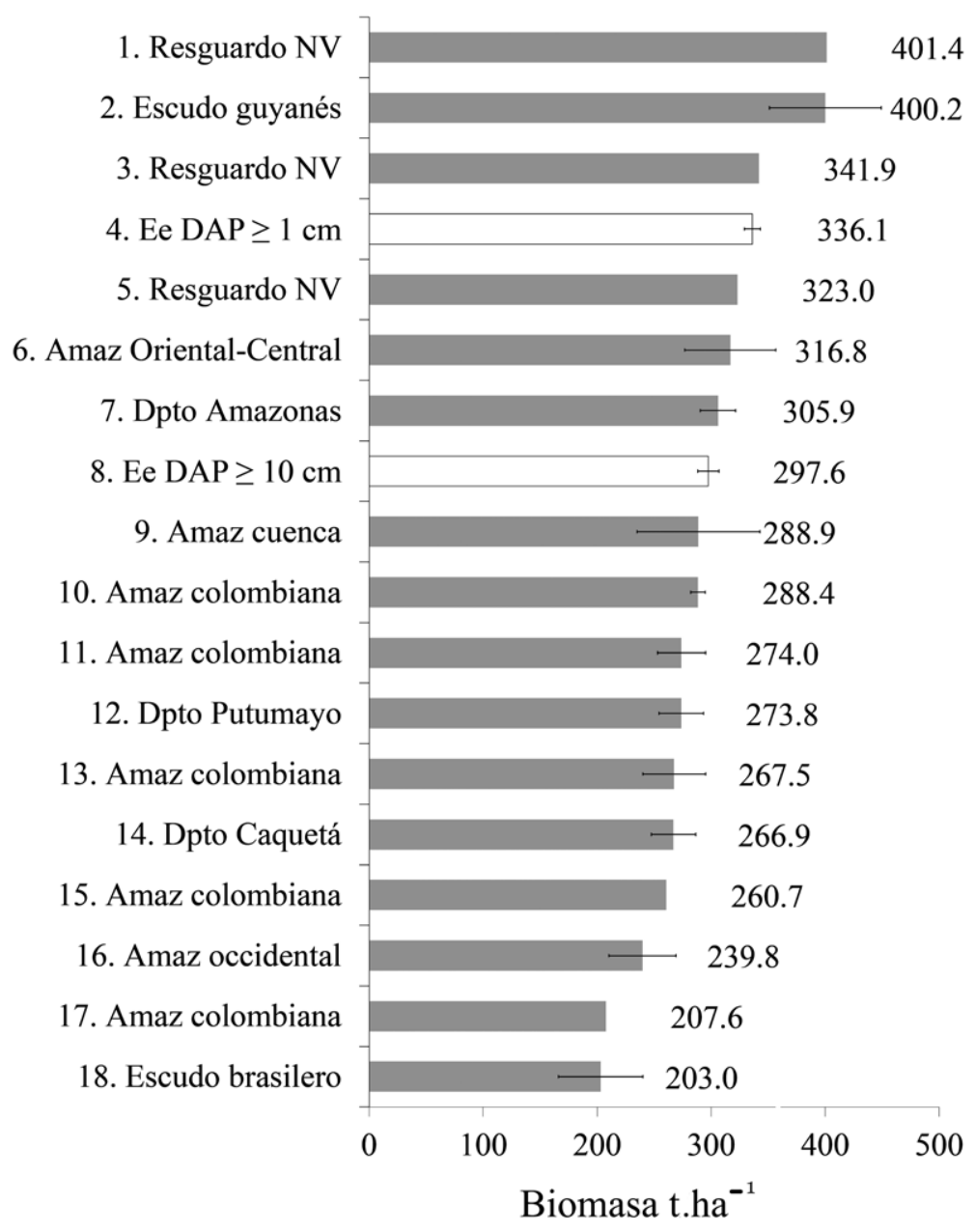

Figura 3. Comparación de la biomasa $\left(\right.$ t.ha $\left.^{-1}\right)$ estimada en este estudio (Ee) para árboles con DAP $\geq 1 \mathrm{~cm}$ y DAP $\geq$ $10 \mathrm{~cm}$, con otros reportes para el medio Caquetá, la Amazonia colombiana y la cuenca Amazónica. Se presentan los valores reportados por los diferentes autores, y su respectiva desviación estándar cuando esta estuvo disponible. Fuente: Álvarez et al. (2017) (13); Anaya et al. (2009) (15); Asner et al. (2012) (17); Baccini et al. (2012) (5); Galindo et al. (2011) (7, 11, 12, 14); Londoño (2011) (3); Mitchard et al. (2014) (2, 6, 9, 16, 18); Phillips et al. (2016) (10); Saatchi et al. (2011) (1). 
respecto a la biomasa encontrada en el presente estudio $\left(297.6 \pm 18.8\right.$ t.ha $\left.^{-1}\right)$, como en el caso de los mapas pantropicales que reportan una alta biomasa entre (323.0 a 401.4 t.ha $^{-1}$ ) y el trabajo de Asner et al. (2012) que reporta un valor relativamente bajo de 207.6 t.ha $^{-1}$, pero similar al valor reportado recientemente para la Amazonia por Phillips et al., (2016) de $288.4 \pm 12.6$ t.ha $^{-1}$. En el contexto de los datos más recientes para la cuenca Amazónica (Mitchard et al. 2014), la biomasa en este estudio es más similar a la de los bosques de Amazonia oriental-central (316.8 \pm 79.7), que a los de Amazonia occidental $\left(239.8 \pm 58.8\right.$ t.ha $\left.^{-1}\right)$.

Los árboles, lianas y palmas del sotobosque almacenaron $11.5 \%$ de la biomasa con respecto al total. Este resultado se corresponde con un estudio en Amazonia central donde se reporta hasta de $9.4 \%$ para árboles (Nascimento \& Laurance, 2002) pero contrasta con otros a nivel mundial que reportan valores promedio de 5.5\% (95\% IC 4.3-6.9) (Vincent et al., 2015). Este promedio para bosques tropicales proviene de los reportes de $4.8 \%$ en Gabón (Memiaghe et al., 2016), entre 2.7-4.2\% en Panamá (Chave et al., 2003; Kirby \& Potvin, 2007), 7.5-7.8\% en la amazonia ecuatoriana (Valencia et al., 2009), 4.58\% en Singapur (Ngo et al., 2013) y 7.2\% en Papua Nueva Guinea (Vincent et al., 2015).

Los resultados contrastan también con otros trabajos que indican que los arboles grandes con $\mathrm{DAP} \geq 70 \mathrm{~cm}$ almacenan gran parte de la biomasa de los bosques tropicales. En nuestro caso, incluso la biomasa del sotobosque fue mayor que la de los árboles grandes. Un resultado similar al del presente estudio fue reportado por Lin et al., (2012) en los bosques subtropicales de hoja perenne de China, donde los árboles de pequeño diámetro aportaron el $10.4 \%$ de la biomasa total sobre el suelo, más que los árboles de $50 \mathrm{~cm}$ de DAP. Diferentes autores atribuyen la baja biomasa de los árboles grandes reportada en algunos sitios a una mayor dinámica del bosque; mayores tasas de mortalidad de árboles dan como resultado un bosque más heterogéneo a escala local donde las áreas recientemente perturbadas contienen mayor número de árboles pequeños que áreas adyacentes. En general, se espera que los bosques más dinámicos almacenen menos carbono en general, tengan mayor variación espacial, menos biomasa en los árboles grandes y proporcionalmente más carbono en los árboles pequeños, en comparación con los bosques menos dinámicos (Vincent et al., 2015). Los estudios a nivel de la cuenca amazónica indican, en general, que en la parte noroccidental, más cerca de los Andes, los suelos son más fértiles y albergan bosques más dinámicos con mayores tasas de mortalidad de árboles y menor biomasa promedio que los de la región central-oriental y los del escudo Guyanés (Quesada et al., 2012) De hecho, Chao et al., (2008) mostraron que los bosques de la Amazonia occidental en el norte de Perú tienen una tasa de mortalidad del $2.5 \%$, mientras que en los bosques del escudo guyanés en la Amazonia oriental es solo del $1.5 \%$. Sin embargo, en el caso del presente estudio la explicación de la alta biomasa del sotobosque puede ser más compleja. En primer lugar, la tasa de mortalidad reportada por Londoño et al., (2011) para el bosque es inusualmente baja ( $1.1 \%$ para un período de 8.6 años) y puede estar relacionada con la poca fertilidad de los suelos y particularmente con el bajo contenido de fosforo, un elemento que determina las tasas de rotación de los árboles a escala de Amazonia (Quesada et al., 2012). En segundo lugar, la baja fertilidad de estos suelos se relaciona principalmente con el material parental altamente lixiviado, que proviene del escudo de Guayana (Duivenvoorden et al., 2005). En este sentido, una alta biomasa de árboles con DAP $\geq 10 \mathrm{~cm}$ en asocio con un reducido número de árboles grandes y una baja rotación de árboles puede estar asociada en nuestro caso con una alta biomasa del sotobosque. Desafortunadamente, los datos de biomasa del sotobosque para la Amazonia son escasos en la literatura científica, con algunas pocas excepciones (Mello et al., 2016; Nascimento \& Laurance, 2002). La ausencia de árboles grandes puede ser una razón que promueve el desarrollo de un sotobosque denso y con alta biomasa, independiente de la dinámica del 
bosque. Por consiguiente, la dinámica de los árboles pequeños puede ser relativamente importante para la función global del ecosistema como lo sugieren algunos autores (Royo \& Carson, 2006), una conclusión opuesta de otros estudios que muestran una contribución mayor de los árboles de gran diámetro (Bastin et al., 2015; LaFrankie et al., 2006; Lutz et al., 2012; Slik et al., 2013).

\section{La heterogeneidad local}

La variación observada a medida que se reduce el tamaño de las subparcelas contiguas, dentro de las 6 ha (figura 3), apoya el concepto de que los bosques maduros son un mosaico constituido por diferentes fases de sucesión y acumulación de biomasa (Chambers et al., 2013). Una consideración importante que debe ser tenida en cuenta cuando se quiere evaluar con precisión el almacenamiento de carbono (Chave et al., 2001; Chave et al., 2003), especialmente a escala del paisaje (Magnabosco-Marra et al., 2016). Algunos autores muestran que un número relativamente pequeño de parcelas permite evaluar con precisión el carbono forestal a escala del paisaje siempre y cuando se distribuyan aleatoriamente (Sierra et al., 2007) y se logre una adecuada representación de la variación espacial den la biomasa (Magnabosco-Marra et al., 2016). Sin embargo, los estudios en parcelas grandes, o relativamente grandes como la del presente estudio en la Amazonia colombiana, muestran que la variación local puede ser tan importante como la regional (Réjou-Méchain et al., 2014; Vincent et al., 2015). En muchos estudios con parcelas pequeñas se presentan sesgos, ya que con frecuencias su localización se elige en función de la presencia de árboles grandes y de una apariencia no perturbada del bosque (Phillips et al., 2002). Las mediciones obtenidas de parcelas pequeñas, cuando se extrapolan a escalas más grandes, pueden afectar dramáticamente las estimaciones de carbono a nivel del paisaje. Recientemente, Réjou-Méchain et al., (2014) demostraron que el error de muestreo en la estimación de la biomasa se incrementa en una magnitud de 1 a 3 cuando se reduce el tamaño de la parcela de 1 ha a 0.1 ha, con grandes implicaciones para la precisión con que se estima el carbono a escala del paisaje. Las parcelas grandes reducen este sesgo y mejoran la precisión ya que integran etapas sucesionales, topografía y otras fuentes de heterogeneidad local que influyen en la estimación de la biomasa (Chave et al., 2003).

\section{Estimación del almacenamiento de carbono en el resguardo Villazul-Nonuya}

Se realizó una extrapolación para los bosques del resguardo Villazul-Nonuya con los diferentes reportes de la literatura para efectos de comparación. El carbono se estimó para todo el resguardo, pero esto tiene algunas restricciones pues además de los bosques de tierra firme sobre el Plano sedimentario terciario en el área existe otra variedad de tipos de bosque (inundables, arenas blancas, etc.), bosques sucesionales y áreas de cultivo (Duivenvoorden, 1995) en una extensión aproximada al 20\% de su área, de acuerdo con información extraída a partir de un mapa digital del resguardo y el mapa de ecosistemas de Colombia (Ideam et al., 2007). Asumiendo esta restricción, para las 260393 ha del resguardo se estimó un contenido de carbono entre 27.1 a 52.4 megatoneladas (Mt) calculadas con el promedio para la amazonia de Asner et al., (2012) y el valor para el sitio obtenido del mapa Pantropical de Saatchi et al., (2011) (tabla 4).

De igual manera, considerando la heterogeneidad espacial de la biomasa dentro de la parcela de 6 ha se obtuvieron valores para los árboles con DAP $\geq 10 \mathrm{~cm}$ entre 37.15 a $40.48 \mathrm{Mt}$, para parcelas de 1 ha, y entre 36.27 a 41.36 Mt para las subparcelas de 0.1 ha. Este ejemplo muestra claramente que la variación espacial y el tamaño de la parcela determinan la incertidumbre de las estimaciones de la biomasa, similar que en otros estudios (Chave et al., 2004). Por tal razón, las estimaciones 
Tabla 4. Estimación del carbono promedio (Prom), con su desviación estándar (De), los valores mínimos (mín) y máximos (máx), almacenado por hectárea y para el área total del resguardo Villazul-Nonuya (RVN) del medio Caquetá, con base en los resultados del presente estudio y otros reportes de la literatura.

\begin{tabular}{|c|c|c|c|c|c|c|c|c|}
\hline \multirow{2}{*}{ Fuente } & \multirow{2}{*}{ Región } & \multicolumn{4}{|c|}{ Carbono t.ha-1 } & \multicolumn{3}{|c|}{ Carbono RVN (Mt) } \\
\hline & & Prom & De & Mín & Máx & Prom & Mín & Máx \\
\hline Galindo et al. (2011) & Amazonas Dpto & 153.0 & 15.5 & 137.5 & 168.4 & 39.9 & 35.9 & 43.9 \\
\hline Galindo et al. (2011) & Amazonas region & 137.0 & 21.0 & 116.0 & 158.0 & 35.7 & 30.3 & 41.2 \\
\hline Galindo et al. (2011) & Caquetá Dpto & 133.4 & 19.5 & 114.0 & 152.9 & 34.8 & 29.7 & 39.9 \\
\hline Galindo et al. (2011) & Putumayo Dpto & 136.9 & 19.5 & 117.4 & 156.4 & 35.7 & 30.6 & 40.8 \\
\hline Phillips et al. (2016) & BhT Col-Amaz & 144.2 & 6.3 & 137.9 & 150.5 & 37.6 & 36.0 & 39.3 \\
\hline Asner et al. (2012) & Amazonia col & 103.8 & & & & 27.1 & & \\
\hline Londoño (2011) & PST RNV & 171.0 & & & & 44.6 & & \\
\hline Álvarez et al. (2017) & Amaz col & 133.8 & 27.6 & 106.2 & 161.3 & 34.9 & 27.7 & 42.1 \\
\hline Baccini et al. (2012) & PST Amaz Col & 161.5 & & & & 42.1 & & \\
\hline Saatchi et al. (2011) & PST Amaz Col & 200.7 & & & & 52.4 & & \\
\hline Anaya et al. (2009) & PST Amaz Col & 130.4 & & & & 34.0 & & \\
\hline Este estudio (total) & PST Amaz Col & 169.6 & 7.5 & 162.2 & 177.1 & 44.3 & 42.3 & 46.2 \\
\hline Este estudio (DAP $\geq 10 \mathrm{~cm})$ & PST RNV & 148.8 & 9.4 & 139.4 & 158.2 & 38.8 & 36.4 & 41.3 \\
\hline
\end{tabular}

de biomasa requeridas por los esquemas dispuestos para incentivar económicamente la preservación del bosque en los programas REDD+ deben ser conservadoras.

Los resultados del presente estudio indican una variación sustancial de la biomasa en un bosque contiguo que normalmente en las estimaciones regionales, nacionales o pantropicales se asumiría como homogéneo. El estimativo final, considerando la biomasa del sotobosque arroja un valor total de 44.3 Mt (rango 42.3 a 46.2 Mt) con base en el área total del reguardo Villazul Nonuya. Considerando que los datos analizados provienen de un bosque de tierra firme, la inclusión de estimativos de biomasa para parcelas establecidas en otros tipos de bosques (inundables, arenas blancas, etc.) evidentemente ayudará a mejorar las estimaciones. Por último, aunque parcelas relativamente grandes como la analizada en este estudio no son prácticas para la implementación de proyectos REDD +, es evidente su importancia para comprender la heterogeneidad espacial local.

\section{CONCLUSIONES}

La medición más detallada de la biomasa en una parcela continua de 6 ha en los bosques de tierra firme de la Amazonia colombiana está en el rango de los valores reportados previamente para la región. Se encontró un alto contenido de biomasa en el sotobosque y una baja contribución de los árboles grandes (DAP $\geq 70 \mathrm{~cm}$ ), más allá de los que se supone para los bosques tropicales de tierras bajas en Amazonia. Se concluye que conocer la variación espacial local es importante y que los árboles pequeños deben ser considerados en futuros estudios de almacenamiento de carbono de los bosques de Amazonia para asegurar que las estimaciones sean más precisas de lo que son actualmente.

\section{AGRADECIMIENTOS}

Este artículo es producto de las redes Rainfor y ColTree y de investigadores de ForestPlots.net. Los autores agradecen el apoyo de la comunidad indígena 
Nonuya-Villazul, quienes aportaron ideas y su propio tiempo para la mayor parte del trabajo de campo; especialmente a Elias Moreno y José Moreno. Los fondos del Convenio Universidad de Leeds y el JBMED, provenientes de una beca de la Fundación Gordon y Betty Moore y de Colciencias en Colombia (Contrato 393 a 2012), financiaron parcialmente el trabajo de campo y oficina. Esteban Álvarez y Edwin Paky fueron financiados por la Fundación ConVida y la Unad (Universidad Nacional Abierta y a Distancia) durante el trabajo de campo 20162017 y durante el tiempo requerido para el análisis de los datos y la escritura del artículo.

\section{CONFLICTO DE INTERESES}

El autor declara no tener conflicto de intereses.

\section{CONTRIBUCIÓN POR AUTOR}

El autor único es responsable de la obra en todos los aspectos que condujeron a la elaboración de su publicación.

\section{REFERENCIAS BIBLIOGRÁFICAS}

Álvarez, E., Duque, A., Saldarriaga, J., Cabrera, K., Salas, G., Valle, I., Lema, A., Moreno, F., Orrego, S. \& Rodríguez, L. (2012). Tree above-ground biomass allometries for carbon stocks estimation in the natural forests of Colombia. Forest Ecology and Management, 267, 297-308.

Álvarez-Dávila, E., Cayuela, L., González-Caro, S., Aldana, A. M., Stevenson, P. R., Phillips, O., CogoIlo, A., Peñuela, M.C., von Hildebrand, P., Jiménez, E., Melo, O., Velasquez, O., Fernández, F., Londoño-Vega, C., Velázquez-Rua, C., Serna, M., Mendoza, I. \& Rey-Benayas, J. M. (2017). Forest Biomass Density across Large Climate Gradients in Northern South America is related to Water Availability but not with Temperature. PLOS ONE 12(3), e0171072. https://doi.org/10.1371/journal.pone.0171072

Anaya, J. A., Chuvieco, E. \& Palacios-Orueta, A. (2009). Aboveground biomass assessment in Colombia: A remote sensing approach. Forest Ecology and Management, 257(4), 1237-1246.

Asner, G. P., Clark, J. K., Mascaro, J., Galindo García, G. A., Chadwick, K. D., Navarrete Encinales, D. A., Paez-Acosta, G., Cabrera Montenegro, E., Kennedy-Bowdoin, T., Duque, Á, Balaji, A., Von Hildebrand, P., Maatoug, L., Phillips Bernal, J. F., Yepes Quintero, A. P., Knapp, D. E., García Dávila, M. C., Jacobson, J. \& Ordóñez, M. F. (2012). High-resolution mapping of forest carbon stocks in the Colombian Amazon. Biogeosciences, 9(7), 2683-2696.

Baccini, a., Goetz, S. J., Walker, W. S., Laporte, N. T., Sun, M., Sulla-Menashe, D., Hackler, J., Beck, P. S., Dubayah, R., Friedl, M., Samanta, S. \& Houghton, R. (2012). Estimated carbon dioxide emissions from tropical deforestation improved by carbon-density maps. Nature Climate Change, 2(3), 182-185.

Bastin, J.-F., Barbier, N., Réjou-Méchain, M., Fayolle, A., Gourlet-Fleury, S., Maniatis, D., Haulleville, T., Baya, F., Beeckman, H., Beina, D., Couteron, P., Chuyong, G., Dauby, G., Doucet, J-L., Droissart, V., Dufrêne, M., Ewango, C., Gillet, J. F., Gonmadje, C. H., Hart, T., Kavali, T., Kenfack, D., Libalah, M., Malhi, Y., Makana, J-R., Pélissier, R., Ploton, P., Serckx, A., Sonké, B., Stevart, T., Thomas, D. W., De Cannière, C. \& Bogaert, J. (2015). Seeing Central African forests through their largest trees. Scientific Reports, 5(august), 1-8.

Bradford, M. G., Metcalfe, D. J., Ford, A., Liddell, M. J. \& McKeown, A. (2014). Floristics, stand structure and aboveground biomass of a 25-ha rainforest plot in the wet tropics of Australia. Journal of Tropical Forest Science, 26(4), 543-553.

Chambers, J. Q. J. Q., Negron-Juarez, R. I. R. I., Marra, D. M. D. M., Di Vittorio, A., Tews, J., Roberts, D., Ribeiro, G., Trumbore, S. \& Higuchi, N. (2013). The steady-state mosaic of disturbance and succession across an old-growth Central Amazon forest landscape. Proceedings of the National Academy of Sciences, 110(10), 3949-3954. 
Chao, K. J., Phillips, O. L., Gloor, E., Monteagudo, A., Torres-Lezama, A. \& Martínez, R. V. (2008). Growth and wood density predict tree mortality in Amazon forests. Journal of Ecology, 96(2), 281-292.

Chave, J., Andalo, C., Brown, S., Cairns, M. A., Chambers, J. Q., Eamus, D., Fölster, H., Fromard, F., Higuchi, N., Kira, T., Lescure, J. P., Nelson, B. W., Ogawa, H., Puig, H., Riéra, B. \& Yamakura, T. (2005). Tree allometry and improved estimation of carbon stocks and balance in tropical forests. Oecologia, 145(1), 87-99.

Chave, J., Condit, R., Aguilar, S., Hernandez, A., Lao, S. \& Perez, R. (2004). Error propagation and scaling for tropical forest biomass estimates. Philosophical Transactions of the Royal Society B: Biological Sciences, 359(1443), 409-420.

Chave, J., Condit, R., Lao, S., Caspersen, J. P., Foster, R. B. \& Hubbell, S. P. (2003). Spatial and temporal variation of biomass in a tropical forest: Results from a large census plot in Panama. Journal of Ecology, 91(2), 240-252.

Chave, J., Réjou-Méchain, M., Búrquez, A., Chidumayo, E., Colgan, M. S., Delitti, W. B. C., Duque, A., Eid, T., Fearnside, P. M., Goodman, R. C., Henry, M., Martínez-Yrízar, A., Mugasha, W. A., MuIler-Landau, H. C., Mencuccini, M., Nelson, B. W., Ngomanda, A., Nogueira, E. M., Ortiz-Malavassi, E., Pélissier, R., Ploton, P., Ryan, C. M., Saldarriaga, J. G. \& Vieilledent, G. (2014). Improved allometric models to estimate the aboveground biomass of tropical trees. Global Change Biology, 20(10), 3177-3190.

Chave, J., Riera, B. \& Dubois, M.-A. (2001). Estimation of biomass in a neotropical forest of French Guiana: spatial and temporal variability. Journal of Tropical Ecology, 17, 79-96.

Duivenvoorden, J. F., Duque, A., Cavelier, J., Garcia, A., Grandez, C., Macia, M. J., Romero-Saltos, H., Sanchez, M. \& Valencia, R. (2005). Density and diversity of plants in relation to soil nutrient reserves in well-drained upland forests in the north-western Amazon basin. Kongelige Danske Videnskabernes Selskab Biologiske Skrifter, 55, 25-35.
Duivenvoorden J.F. (1995). Tree species composition and rainforest-environmental relationship in the middle Caquetá area Colombia, NW Amazonia. Vegetation. Vegetatio, 120(2), 91-113.

Eggleston HS, Buendia L, Miwa K, Ngara T \& Tanabe, K. (eds). (2006). 2006 IPCC guidelines for national greenhouse gas inventories. Japón: Task Force on National Greenhouse Gas Inventories (TFI) of the IPCCIGES. Fecha de acceso: 08 de noviembre de 2011. Recuperado de: http://www.ipcc-nggip.iges.tor.jp/ public/2006gl/pdf/O_Overview/V0_0_Cover.pdf

Fauset, S. et al. (2015). Hyperdominance in Amazonian forest carbon cycling. Nature Communications, 6, 6857.

Feldpausch, T. R. et al. (2012). Tree height integrated into pantropical forest biomass estimates. Biogeosciences, 9(8), 3381-3403.

Galindo, G., Cabrera, E., Vargas, D., Pabón, H., Yepes, A., Phillips, J. F., Navarrete, D., Duque, A. García, M. \& Ordoñez, M. F. (2011). Estimación de la biomasa aérea usando datos de campo e información de sensores remotos Versión 1.0. Bogotá: Ideam. $50 p$.

García-Arbeláez, C., Barrera, X., Gómez, R. \& Suárez-Castaño, R. (2015). El ABC de los compromisos de Colombia para la Cop 21. Bogotá: WWF-Colombia.

Goodman, R. C., Phillips, O. L., Del Castillo Torres, D., Freitas, L., Cortese, S. T., Monteagudo, A. \& Baker, T. R. (2013). Amazon palm biomass and allometry. Forest Ecology and Management, 310, 994-1004.

Holdridge, R. (1982). Ecología basada en zonas de vida. San José de Costa Rica: Instituto Interamericano de Cooperación para la Agricultura (IICA). 216 pp.

Instituto de Hidrología, Meteorología y Estudios Ambientales (Ideam), Instituto Geográfico Agustín Codazzi (Igac), Instituto de Investigaciones Marinas y Costeras "José Benito Vives de Andreis" (Invemar), Instituto Amazónico de Investigaciones científicas (Sinchi) \& Instituto de Investigaciones Ambientales del Pacífico (IIAP) (2007). Mapa de Ecosistemas Continentales. Costeros y Marinos de Colombia. Bogotá: Ideam. 
Kirby, K. R. \& Potvin, C. (2007). Variation in carbon storage among tree species: Implications for the management of a small-scale carbon sink project. Forest Ecology and Management, 246(2-3), 208-221.

LaFrankie, J. V., Ashton, P. S., Chuyong, G. B., Co, L., Condit, R., Davies, S. J., Foster, R., Hubbell, S., Kenfack, D., Lagunzad, D., Losos, E., Nor, N., Tan, S., Thomas, D., Valencia, R. \& Villa, G. (2006). Contrasting structure and composition of the understory in species-rich tropical rain forests. Ecology, 87(9), 2298-2305.

Larson, A. M., Corbera, E., Cronkleton, P., Van Dam, C., Bray, D. B., Estrada, M., ... \& Pacheco, P. (2010). Rights to forests and carbon under REDD+ initiatives in Latin America. Bogor, Indonesia: Center for International Forestry Research (CIFOR). 8 p.

Lin, D., Lai, J., Muller-Landau, H. C., Mi, X. \& Ma, K. (2012). Topographic Variation in Aboveground Biomass in a Subtropical Evergreen Broad-Leaved Forest in China. PLoS ONE, 7(10), 22-24.

Londoño, A. C. (2011). Flora and dynamics of an upland and a floodplain forest in Peña Roja, Colombian Amazonia (Ph.D. thesis). Amsterdam: Universiteit van Amsterdam. 243 p.

Londoño, A. C. \& Alvarez, E. (1997). Composicion floristica de dos bosques ( tierra firme y varzea ) en la region de araracuara, amazonia colombiana. Caldasia, 19(3), 431-463.

Londoño, A. C., Alvarez, E., Forero, E. \& Morton, C. M. (1995). A New Genus and Species of Dipterocarpaceae from the Neotropics. I. Introduction, Taxonomy, Ecology, and Distribution. Brittonia, 47(3), 225-236.

Lutz, J. A., Larson, A. J., Swanson, M. E. \& Freund, J. A. (2012). Ecological importance of large-diameter trees in a temperate mixed-conifer forest. PLoS ONE, 7(5). http://doi.org/10.1371/journal. pone.0036131

Magnabosco-Marra, D., Higuchi, N., Trumbore, S. E., Ribeiro, G. H. P. M., Dos Santos, J., Carneiro, V. M. C., Lima, A., Chambers, J., Negrón-Juárrez, R., Holzwarth, F., Reu, B. \& Wirth, C. (2016). Predicting biomass of hyperdiverse and structurally complex central Amazonian forests-A virtual approach using extensive field data. Biogeosciences, 13(5), 1553-1570.

Malhi, Y., Baker, T. R., Phillips, O. L., Almeida, S., Alvarez, E., Arroyo, L., Chave, J., Czimczik, C., Di Fiore, A., Higuchi, N., Killeen, T., Laurance, S., Laurance, W., Lewis, S., Mercado-Montoya, L., Monteagudo, A., Neill, D., Vargas, P., Patino, S., Pitman, N., Quesada, C., Salomao, R., Silva, J., Lezama, A., Martínez, R., Terborgh, J., Vinceti, B. \& Lloyd, J. (2004). The above-ground coarse wood productivity of 104 Neotropical forest plots. Global Change Biology, 10, 563-591.

Mello, L. N. do C., Sales, M. H. R. \& Rosa, L. P. (2016). Analysis of results of biomass forest inventory in northeastern amazon for development of REDD+ carbon project. Anais Da Academia Brasileira de Ciencias, 88(1), 55-64.

Memiaghe, H. R., Lutz, J. A., Korte, L., Alonso, A. \& Kenfack, D. (2016). Ecological Importance of SmaII-Diameter Trees to the Structure, Diversity and Biomass of a Tropical Evergreen Forest at Rabi, Gabon. PLoS ONE, 11(5), 1-15.

Mitchard, E. T. et al. (2014). Markedly divergent estimates of Amazon forest carbon density from ground plots and satellites. Global Ecology and Biogeography, 23, 935-946.

Murphy, H. T., Bradford, M. G., Dalongeville, A., Ford, A. J. \& Metcalfe, D. J. (2013). No evidence for long-term increases in biomass and stem density in the tropical rain forests of Australia. Journal of ECOlogy, 101(6), 1589-1597.

Nascimento, H. E. M. \& Laurance, W. F. (2002). Total aboveground biomass in central Amazonian rainforests: A landscape-scale study. Forest Ecology and Management, 168(1-3), 311-321.

Ngo, K. M., Turner, B. L., Muller-Landau, H. C., Davies, S. J., Larjavaara, M., Nik Hassan, N. F. bin \& Lum, S. (2013). Carbon stocks in primary and secondary tropical forests in Singapore. Forest Ecology and Management, 296, 81-89.

Phillips, J., Duque, A., Scott, C., Wayson, C., Galindo, G., Cabrera, E.,Chave, J., Peña, M., Alvarez, E., Carrdenas, D., Duivenvoorden, J., Hildebrand, P., Stevenson, P., Ramírez, S. \& Yepes, A. (2016). Live 
aboveground carbon stocks in natural forests of $\mathrm{CO}-$ lombia. Forest Ecology and Management, 374(August), 119-128.

Phillips, J., Duque, A., Yepez, A., García, M., Navarrete, D. A., Álvarez, E. \& Cárdenas, D. (2011). Estimación de las reservas actuales (2010) de carbono almacenadas en la biomasa aérea en bosques naturales de Colombia-Estratificación, alometría y métodos análiticos. Bogotá: Ideam.

Phillips, O., Malhi, Y., Vinceti, B., Baker, T., Lewis, S., Higuchi, N., Laurance, W. F., Núñez Vargas, P., Vásquez Martínez, R., Laurance, S., Ferreira, L. V., Stern, M., Brown, S. \& Grace, J. (2002). Changes in growth of tropical forests: evaluating potential biases. Ecological Applications, 12(2), 576-587.

Quesada, C.A., Phillips, O.L., Schwarz, M., Czimczik, C.I., Baker, T.R. \& Patiño, S.(2012). Basin-wide variations in Amazon forest structure and function are mediated by both soils and climate. Biogeosciences, 9, 2203-2246.

Réjou-Méchain, M. et al. (2014). Local spatial structure of forest biomass and its consequences for remote sensing of carbon stocks. Biogeosciences, 11(23), 6827-6840.

Rodriguez, L. (1991). Biomasa y reserva de nutrientes en un ecosistema de tierra firme en la región de Araracuara. Bogotá: Fundación Tropenbos-Colombia. Documento sin publicar.

Royo, A. \& Carson, W. P. (2006). On the formation of dense understory layers in forests worldwide: consequences and implications for forest dynamics, biodiversity, and succession. Canadian Journal of Forest Research, 36(6), 1345-1362.

Saatchi, S. S., Harris, N. L., Brown, S., Lefsky, M., Mitchard, E. T. , Salas, W., Zutta, B. R., Buermann, W., Lewis, S. L., Hagen, S., Petrova, S., White, L., Silman, M. \& Morel, A. (2011). Benchmark map of forest carbon stocks in tropical regions across three continents. Proceedings of the National Academy of Sciences of the United States of America, 108, 9899-904.

Salinas-Abdala, Y. (2014). Los derechos territoriales de los grupos étnicos: ¿un compromiso social, una obligación constitucional o una tarea hecha a medias? Punto de Encuentro, 67, 1-39.

Schnitzer, S. A., DeWalt, S. J. \& Chave, J. (2006). Censusing and measuring lianas: A quantitative comparison of the common methods. Biotropica, 38(5), 581-591.

Sierra, C. A., del Valle, J. I., Orrego, S. A., Moreno, F. H., Harmon, M. E., Zapata, M., Colorado, G. J., Herrera, M. A., Lara, W., Restrepo, D. E., Berrouet, L. M., Loaiza, L. M. \& Benjumea, J. F. (2007). Total carbon stocks in a tropical forest landscape of the Porce region, Colombia. Forest Ecology and Management, 243(2-3), 299-309.

Slik, J. W. F. et al. (2013). Large trees drive forest aboveground biomass variation in moist lowland forests across the tropics. Global Ecology and Biogeography, 22(12), 1261-1271.

Statgraphics Centurion, X. V. I. (2013). Statgraphics centurion XVI software version 16.2. 04. Virginia (USA): . StatPoint Technologies Inc, Warrenton. Recuperado de: www.statgraphics.net.

Valencia, R., Condit, R., Muller-Landau, H. C., Hernandez, C. \& Navarrete, H. (2009). Dissecting biomass dynamics in a large Amazonian forest plot. Journal of Tropical Ecology, 25(5), 473.

Vincent, J. B., Henning, B., Saulei, S., Sosanika, G. \& Weiblen, G. D. (2015). Forest carbon in lowland Papua New Guinea: Local variation and the importance of small trees. Austral Ecology, 40(2), 151-159.

Watch, C. T. (2013). Protecting Carbon to destroy forests. Land enclosures and REDD+. Carbon Trade Watch. Published by TNI, FDCL and IGO for the Hands off the Land Alliance. Recuperado de: http:// www.carbontradewatch.org/downloads/publications/REDD_and_land-web.pdf.

Zanne, A. E., Lopez-Gonzalez, G., Coomes, D. A., Ilic, J., Jansen, S., Lewis, S. L., Miller, R. B., Swenson, N. G., Wiemann, M. C. \& Chave, J. (2009). Data from: Towards a worldwide wood economics spectrum. Leeds (UK): Dryadlab.org. Recuperado de: http:// dx.doi.org/10.5061/dryad.234

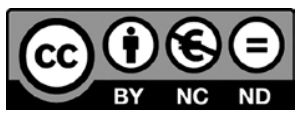

